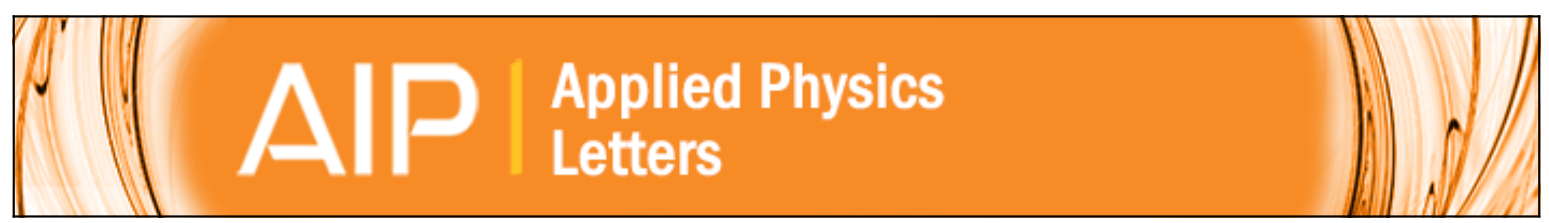

\title{
Upper limit for the modulation bandwidth of a quantum dot laser
}

Levon V. Asryan and Robert A. Suris

Citation: Applied Physics Letters 96, 221112 (2010); doi: 10.1063/1.3446968

View online: http://dx.doi.org/10.1063/1.3446968

View Table of Contents: http://scitation.aip.org/content/aip/journal/apl/96/22?ver=pdfcov

Published by the AIP Publishing

\section{Articles you may be interested in}

Effect of excited states on the ground-state modulation bandwidth in quantum dot lasers

Appl. Phys. Lett. 102, 191102 (2013); 10.1063/1.4804994

Effect of internal optical loss on the modulation bandwidth of a quantum dot laser

Appl. Phys. Lett. 100, 131106 (2012); 10.1063/1.3697683

Carrier capture delay and modulation bandwidth in an edge-emitting quantum dot laser Appl. Phys. Lett. 98, 131108 (2011); 10.1063/1.3571295

Theoretical study on high-speed modulation of Fabry-Pérot and distributed-feedback quantum-dot lasers: $\mathrm{K}$ factor-limited bandwidth and $10 \mathrm{Gbit} s$ eye diagrams

J. Appl. Phys. 101, 013108 (2007); 10.1063/1.2407259

Single-mode submonolayer quantum-dot vertical-cavity surface-emitting lasers with high modulation bandwidth

Appl. Phys. Lett. 89, 141106 (2006); 10.1063/1.2358114

\section{AlP Re-register for Table of Content Alerts}




\title{
Upper limit for the modulation bandwidth of a quantum dot laser
}

\author{
Levon V. Asryan ${ }^{1, a)}$ and Robert A. Suris ${ }^{2, b)}$ \\ ${ }_{2}^{1}$ Virginia Polytechnic Institute and State University, Blacksburg, Virginia 24061, USA \\ ${ }_{2}^{2}$ Ioffe Physico-Technical Institute, Saint Petersburg 194021, Russia
}

(Received 25 April 2010; accepted 17 May 2010; published online 4 June 2010)

\begin{abstract}
We derive a closed-form expression for the upper limit for the modulation bandwidth of a semiconductor quantum dot (QD) laser. The highest possible bandwidth increases directly with overlap integral of the electron and hole wave functions in a QD, number of QD-layers, and surface density of QDs in a layer, and is inversely proportional to the inhomogeneous line broadening caused by the QD-size dispersion. At 10\% QD-size fluctuations and 100\% overlap, the upper limit for the modulation bandwidth in a single QD-layer laser can be as high as $60 \mathrm{GHz}$. () 2010 American Institute of Physics. [doi:10.1063/1.3446968]
\end{abstract}

Due to the capability of direct modulation of the optical output by electric current, semiconductor lasers are extensively used in high-speed fiber networks. In quantum dot (QD) lasers, the stimulated emission is produced in nanosize regions confining the charge carriers in all three spatial dimensions. ${ }^{1}$ A discrete energy spectrum of carriers in QDs enables lasing with low threshold current ${ }^{2-4}$ and high temperature-stability. ${ }^{5}$ The modulation bandwidth of QD lasers, however, needs to be enhanced.

In this work, we study the modulation bandwidth of semiconductor lasers with a quantum-confined active region. Although our analysis and derivations are general and apply also to quantum well and quantum wire lasers, our focus here is on a QD laser. We estimate the highest possible intrinsic bandwidth attainable in a coupled electron-photon system of a laser. For this purpose, we do not consider a transport delay across the optical confinement layer (OCL) and a capture delay from the OCL to the active region. For the same purpose, we do not also consider the gain compression with increasing optical power.

We use the small-signal analysis of rate equations. ${ }^{6-12}$ While we assume instantaneous carrier injection into the active region, our model includes the carrier population and recombination in the OCL. In the simplest model, three equations are used-for carriers outside the active region (in the OCL), those in the active region, and photons. The assumptions of no transport and capture delay effectively reduce the number of equations to two. These equations are

$$
\begin{aligned}
& \frac{\partial}{\partial t}(\delta n)=\frac{\delta j}{e b}-\delta R_{\text {non-stim }}-\delta R_{\text {stim }} \\
& \frac{\partial}{\partial t}\left(\frac{\delta N}{V}\right)=\delta R_{\text {stim }}-\delta R_{\text {loss }}
\end{aligned}
$$

where $\delta(\ldots)$ means a small variation of $(\ldots), n=n_{\mathrm{act}}+n_{\mathrm{OCL}}$ is the total carrier density (including the active region and OCL), $j$ is the injection current density, $N$ is the number of photons in the lasing mode, and $b$ and $V$ are the OCL thickness and volume, respectively, $R_{\text {non-stim }}$ is the total rate of nonstimulated recombination processes (including the active

\footnotetext{
${ }^{a)}$ Electronic mail: asryan@mse.vt.edu.

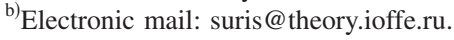

region and OCL), $R_{\text {stim }}$ is the stimulated recombination rate, and $R_{\text {loss }}$ is the photon loss rate.

Assuming a small time-harmonic ac injection current density $\delta j=\delta j_{\mathrm{m}} \exp (i \omega t)$ and correspondingly looking for the solutions of Eqs. (1) and (2) in the form of $\delta n$ $=\delta n_{\mathrm{m}}(\omega) \exp (i \omega t)$ and $\delta N=\delta N_{\mathrm{m}}(\omega) \exp (i \omega t)$, we obtain for the modulation response function

$$
H(\omega)=\left|\frac{\delta N_{\mathrm{m}}(\omega)}{\delta N_{\mathrm{m}}(0)}\right|^{2}=\frac{\omega_{0}^{4}}{\left(\omega^{2}-\omega_{0}^{2}\right)^{2}+4 \Gamma_{\mathrm{dec}}^{2} \omega^{2}} .
$$

The shape of $H(\omega)$ depends strongly on the dc component $j_{0}$ of the injection current density. For a certain range of values of $j_{0}$ (see below), $H(\omega)$ has a peak (Fig. 1) obtained at

$$
\omega_{\text {peak }}=\sqrt{\Omega_{\mathrm{osc}}^{2}-\Gamma_{\mathrm{dec}}^{2}}=\sqrt{\omega_{0}^{2}-2 \Gamma_{\mathrm{dec}}^{2}},
$$

where $\Omega_{\mathrm{osc}}$ and $\Gamma_{\mathrm{dec}}$ are the angular frequency and decay rate of relaxation oscillations,

$$
\begin{aligned}
& \Omega_{\mathrm{osc}}=\sqrt{\omega_{0}^{2}-\Gamma_{\mathrm{dec}}^{2}}, \\
& \Gamma_{\mathrm{dec}}=\frac{1}{2}\left(\frac{1}{\tau_{\text {non-stim }}^{\text {dif }}}+\mathrm{v}_{\mathrm{g}} \frac{G^{\mathrm{dif}}}{V} N_{0}\right),
\end{aligned}
$$

and $\omega_{0}$ is given as

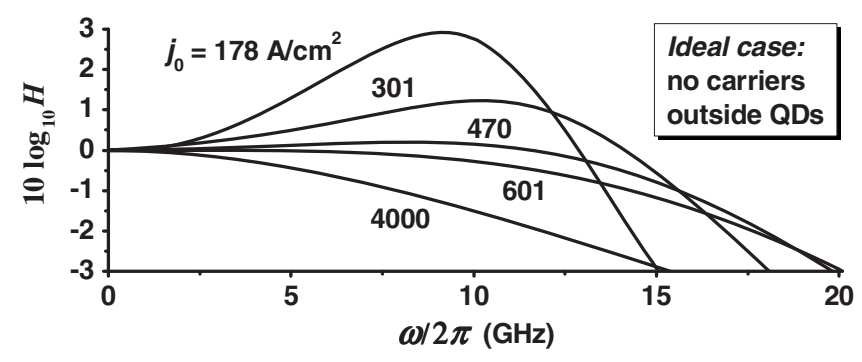

FIG. 1. Response function in the ideal case of no carriers outside QDs at different values of the dc component of the injection current density. In Figs. $1-4$, a GaInAsP structure of Ref. 13 lasing at $T=300 \mathrm{~K}$ near $1.55 \mu \mathrm{m}$ is considered. $Z_{\mathrm{L}}=1,(\Delta \varepsilon)_{\text {inhom }}=7 \mathrm{meV}\left(10 \%\right.$ QD-size fluctuations), $N_{\mathrm{S}}$ $=6.11 \times 10^{10} \mathrm{~cm}^{-2}$, and $I_{\text {overlap }}=1 ; \quad g^{\max }=29.52 \mathrm{~cm}^{-1}$ and $G_{\text {act }}^{\text {dif }}=1.36$ $\times 10^{-14} \mathrm{~cm}^{2}$. The cavity length $L=1.139 \mathrm{~mm}$. At these parameters, $\omega_{-3 \mathrm{~dB}}^{\max } / 2 \pi=20 \mathrm{GHz}$ and $j_{\mathrm{opt}}=601 \mathrm{~A} / \mathrm{cm}^{2}$ (see also Fig. 2). 


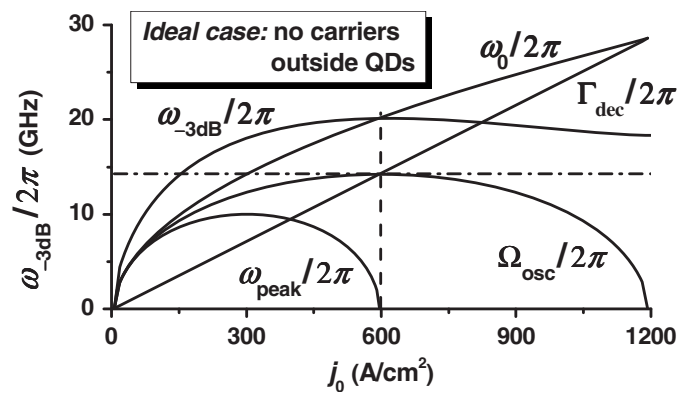

FIG. 2. Modulation bandwidth $\omega_{-3 \mathrm{~dB}} / 2 \pi$, peak frequency of the response function $\omega_{\text {peak }} / 2 \pi$, relaxation oscillation frequency $\Omega_{\text {osc }} / 2 \pi, \Gamma_{\text {dec }} / 2 \pi$, and $\omega_{0} / 2 \pi$ vs dc component of the injection current density in the ideal case of no carriers outside QDs. The vertical dashed line marks $j_{\mathrm{opt}}$.

$$
\omega_{0}=\sqrt{\mathrm{v}_{\mathrm{g}} \frac{G^{\mathrm{dif}}}{V} N_{0} \frac{1}{\tau_{\mathrm{ph}}}} .
$$

In the above equations, $\mathrm{v}_{\mathrm{g}}$ is the group velocity of light, $N_{0}$ is the dc number of photons in the lasing mode, which is related to $j_{0}$ by the steady-state light-current characteristic, $\tau_{\mathrm{ph}}=\left(L / \mathrm{v}_{\mathrm{g}}\right) / \ln (1 / R)$ is the photon lifetime in the cavity, $L$ is the cavity length, and $R$ is the mirror reflectivity.

The effective differential gain $G^{\text {dif }}$ is calculated as the derivative of the modal gain $g$ with respect to the total carrier density $n=n_{\text {act }}+n_{\mathrm{OCL}}$,

$$
G^{\mathrm{dif}}=\frac{\partial g}{\partial n}=\frac{\partial n_{\mathrm{act}}}{\partial n} G_{\mathrm{act}}^{\mathrm{dif}} \ll G_{\mathrm{act}}^{\mathrm{dif}},
$$

where $G_{\text {act }}^{\mathrm{dif}}=\partial g / \partial n_{\text {act }}$. Due to the fact that $\partial n_{\text {act }} / \partial n \ll 1, G^{\mathrm{dif}}$ $\ll G_{\text {act }}^{\text {dif }}$ and, as discussed below, the practically achievable modulation bandwidth of the laser can be significantly reduced.

In Eq. (6), the effective differential non-stimulated recombination time $\tau_{\text {non-stim }}^{\text {dif }}=\left(\partial R_{\text {non-stim }} / \partial n\right)^{-1}$ is expressed in terms of such times in and outside the active region.

The most important dynamic characteristic is the $-3 \mathrm{~dB}$ bandwidth (referred to as the modulation bandwidth $\omega_{-3 \mathrm{~dB}} / 2 \pi$ here) - the frequency at which the response function $H(\omega)$ has fallen to half its $\mathrm{dc}(\omega=0)$ value. The equation for $\omega_{-3 \mathrm{~dB}}$ is

$$
\omega_{-3 \mathrm{~dB}}=\sqrt{\omega_{\text {peak }}^{2}+\sqrt{\omega_{\text {peak }}^{4}+(r-1) \omega_{0}^{4}}},
$$

where $r=10^{0.3} \approx 1.995$.

The relaxation oscillations are only possible $\left[\Omega_{\text {osc }}\right.$ should be real—see Eq. (5)] for a certain range of values of $N_{0}$, i.e., of dc component $j_{0}$ of the injection current density. For such $j_{0}, \Omega_{\text {osc }}$ increases from zero, approaches its maximum value, and then decreases to zero (Fig. 2). The peak of the response function also exists for its own range of $j_{0}\left[\omega_{\text {peak }}\right.$ should be real-see Eq. (4)]; $\omega_{\text {peak }}$ behaves similarly to $\Omega_{\text {osc }}$ with increasing $j_{0}$ (Fig. 2) - what this means is, at a certain $j_{0}$, the peak of $H(\omega)$ appears at $\omega_{\text {peak }}=0$, then it moves to higher frequencies with increasing $j_{0}$, and then moves back to lower frequencies and finally disappears at $\omega_{\text {peak }}=0$ (Fig. 2). At $j_{0}$ value, at which the peak of $H(\omega)$ disappears, $\Omega_{\text {osc }}$ is maximum (Fig. 2).

As a function of $j_{0}$, the modulation bandwidth also has a maximum (Fig. 2). The maximum of $\omega_{-3 \mathrm{~dB}}$ is obtained at approximately the same value $j_{\text {opt }}$ of $j_{0}$, at which the peak of the response function disappears (Fig. 2) At $j_{0}=j_{\text {opt }} H(\omega)$ is most flat (Fig. 1); although $\Omega_{\mathrm{osc}}$ is maximum at $j_{0}=j_{\mathrm{opt}}$, the relaxation oscillations are strongly damped at this current $\left(\Gamma_{\mathrm{dec}}=\Omega_{\mathrm{osc}}^{\max } \approx 1 / \tau_{\mathrm{ph}}\right.$-see Fig. 2). On further increase in $j_{0}$ beyond $j_{\mathrm{opt}}, \omega_{-3 \mathrm{~dB}}$ decreases and asymptotically approaches its saturation value $\left.\omega_{-3 \mathrm{~dB}}\right|_{j_{0} \rightarrow \infty} \approx 1 / \tau_{\mathrm{ph}}$. The maximum values of $\omega_{\text {peak }}, \Omega_{\text {osc }}$, and $\omega_{-3 \mathrm{~dB}}$ are all controlled by the reciprocal photon lifetime in the cavity,

$$
\omega_{-3 \mathrm{~dB}}^{\max } \approx \sqrt{2} \Omega_{\mathrm{osc}}^{\max } \approx \sqrt{2}\left(\sqrt{2} \omega_{\text {peak }}^{\text {max }}\right) \approx \frac{\sqrt{2}}{\tau_{\mathrm{ph}}}=\sqrt{2} \frac{\mathrm{v}_{\mathrm{g}}}{L} \ln \frac{1}{R} .
$$

The shorter the cavity, the higher $\omega_{-3 \mathrm{~dB}}^{\max }$. The shortest cavity length $L^{\mathrm{min}}$ is controlled by the maximum modal gain $g^{\max }$ through the lasing condition (equality of the loss to the gain),

$$
\frac{1}{L^{\min }} \ln \frac{1}{R}=g^{\max } .
$$

Using $L^{\min }$ for $L$ in Eq. (10), we find that the highest possible bandwidth increases directly with $g^{\max }$ and is not affected by the differential gain $G^{\mathrm{dif}}$,

$$
\omega_{-3 \mathrm{~dB}}^{\text {highest }}=\sqrt{2} \mathrm{v}_{\mathrm{g}} g^{\max } \text {. }
$$

While $\omega_{-3 \mathrm{~dB}}^{\text {highest }}$ is controlled by merely $g^{\max }$, and $\omega_{-3 \mathrm{~dB}}^{\max }$ by $L$, the optimum current densities $j_{\mathrm{opt}}$, at which they are obtained, are controlled by $G^{\mathrm{dif}}$ as well. Indeed, the condition for the maximum bandwidth $\left(\omega_{\text {peak }}=0\right)$ is obtained when $\omega_{0}^{2}=2 \Gamma_{\mathrm{dec}}^{2}$ - see Eq. (4). As seen from Eqs. (6) and (7), both $\omega_{0}$ and $\Gamma_{\text {dec }}$ are controlled by the product of $N_{0}$ and $G^{\mathrm{dif}}$. Hence, the lower $G^{\text {dif }}$, the higher will be the photon number $N_{0}=N_{0}^{\text {opt }}$, at which $\omega_{0}^{2}=2 \Gamma_{\text {dec }}^{2}$, i.e., the higher will be $j_{\text {opt }}$. As a result, the practically achievable bandwidth will be reduced compared to Eq. (12) and even Eq. (10).

The above analysis and equations are general and apply to semiconductor lasers with any type of a quantum-confined active region assuming that the carrier transport to and exchange with the latter are instantaneous. In what follows, we focus on QD lasers. Using the expression for $g^{\max }$ for a QD laser, ${ }^{13,14}$ we obtain from Eq. (12)

$$
\begin{aligned}
\frac{1}{2 \pi} \omega_{-3 \mathrm{~dB}}^{\text {highest }}= & \frac{2}{3} \sqrt{2} \xi \frac{\alpha}{\sqrt{\epsilon_{\mathrm{g}}}}\left(\frac{P}{\hbar}\right)^{2}\left(\frac{\lambda_{0}}{\sqrt{\epsilon}} \frac{1}{\mathcal{L}}\right) \\
& \times I_{\text {overlap }} Z_{\mathrm{L}} N_{\mathrm{S}} \frac{\hbar}{(\Delta \varepsilon)_{\text {inhom }}},
\end{aligned}
$$

where $\xi=1 / \sqrt{2 \pi}$ or $1 / \pi$ for Gaussian or Lorentzian QD-size distributions, respectively, $\alpha=e^{2} / \hbar c$ is the fine structure constant, $\sqrt{\epsilon_{\mathrm{g}}}$ and $\sqrt{\epsilon}$ are the group and refractive indices of the dispersive OCL material, $P$ is Kane's parameter $[P / \hbar$ has the dimension of a velocity-see Eq. (3) in Ref. 13], $\lambda_{0}$ is the lasing wavelength, $\mathcal{L}$ is the characteristic length of the light confinement in the transverse direction in the waveguide [see Eq. (9) in Ref. 15], $I_{\text {overlap }}$ is the overlap integral of the electron and hole wave functions in a $\mathrm{QD}, Z_{\mathrm{L}}$ is the number of QD-layers, $N_{\mathrm{S}}$ is the surface density of QDs in one layer, and $(\Delta \varepsilon)_{\text {inhom }}$ is the inhomogeneous line broadening (measured in units of energy) caused by the QD-size dispersion.

The absence of carriers in the OCL would be the bestcase scenario not only for the threshold and power characteristics but for the modulation characteristics as well. For such an ideal case Figs 1 and 2 show the response function 


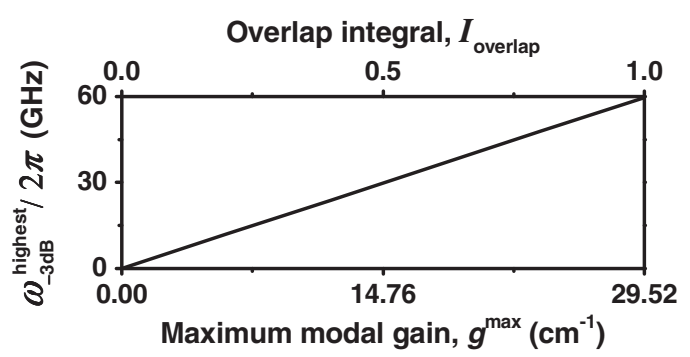

FIG. 3. The upper limit for the modulation bandwidth of a single QD-layer laser vs maximum modal gain and overlap integral of the electron and hole wave functions in a QD.

and the modulation bandwidth $\omega_{-3 \mathrm{~dB}} / 2 \pi$ versus de current density $j_{0}$. The parameters of a specific structure used for an illustration of the results are presented in the caption to Fig. 1. As seen from the figures, $\omega_{-3 \mathrm{~dB}}^{\max }$ is attained at a fairly low $j_{\text {opt }}\left(601 \mathrm{~A} / \mathrm{cm}^{2}\right)$. This is because $G^{\mathrm{dif}}$ is high in this case: $G^{\mathrm{dif}}=G_{\mathrm{act}}^{\mathrm{dif}}=1.36 \times 10^{-14} \mathrm{~cm}^{2}$.

Figure 3 shows the upper limit for the modulation bandwidth $\omega_{-3 \mathrm{~dB}}^{\text {highest }} / 2 \pi$ versus maximum modal gain $g^{\text {max }}$ in a single QD-layer laser. The top axis illustrates the situation when $g^{\max }$ is varied through changing $I_{\text {overlap. }}$ At $10 \%$ QDsize fluctuations $\left[(\Delta \varepsilon)_{\text {inhom }}=7 \mathrm{meV}\right], N_{\mathrm{S}}=6.11 \times 10^{10} \mathrm{~cm}^{-2}$, and ideal overlap of the electron and hole wave functions in a QD $\left(I_{\text {overlap }}=1\right)$, the maximum gain is $g^{\max }=29.52 \mathrm{~cm}^{-1}$, and the shortest cavity length is $L^{\mathrm{min}}=386 \mu \mathrm{m}$. At these parameters, the highest possible modulation bandwidth in a single QD-layer laser is

$$
\omega_{-3 \mathrm{~dB}}^{\text {highest }} / 2 \pi \approx 60 \mathrm{GHz} \text {. }
$$

If the overlap is poor or the QD-size dispersion is large, $g^{\max }$ will be low and so will be $\omega_{-3 \mathrm{~dB}}^{\text {hight }} / 2 \pi$. Thus, $g^{\max }$ $=4.4 \mathrm{~cm}^{-1}$ and $\omega_{-3 \mathrm{~dB}}^{\text {highest }} / 2 \pi \approx 9 \mathrm{GHz}$ if $I_{\text {overlap }}=0.15$. One way to increase $I_{\text {overlap }}$ is the use of more symmetrical (e.g., truncated or disk-shape) QDs. ${ }^{14}$

As seen from Eq. (13), the use of multiple layers with QDs can effectively enhance the modulation bandwidth thus compensating for a poor overlap of the electron and hole wave functions in a QD or for a large QD-size dispersion. The use of submonolayer QDs was also reported to allow for a higher surface density $N_{\mathrm{S}}$ of QDs in a layer. ${ }^{16}$

In the presence of carriers in the OCL, all the above expressions hold. At the same time, $G^{\mathrm{dif}}$ [see Eq. (8)] is considerably reduced as compared to $G_{\text {act }}^{\text {dif }}$. Due to this, $\omega_{-3 \mathrm{~dB}}^{\max }$ (and the more so $\omega_{-3 \mathrm{~dB}}^{\text {hight }}$ ) becomes unattainable at practical values of the pump current density in a single QD-layer laser. In such a specific laser structure considered here, $\partial n_{\text {act }} / \partial n$ $=0.009$, i.e., $G^{\text {dif }}$ is about two orders of magnitude lower than $G_{\text {act }}^{\text {dif }}\left[\right.$ see Eq. (8)]: $G^{\text {dif }}=1.27 \times 10^{-16} \mathrm{~cm}^{2}$. Hence, the photon number $N_{0}=N_{0}^{\mathrm{opt}}$, at which $\omega_{-3 \mathrm{~dB}}^{\max }$ is obtained [see the discussion following Eq. (12)], is two orders of magnitude higher than in the ideal case of no carriers in the OCL, and so

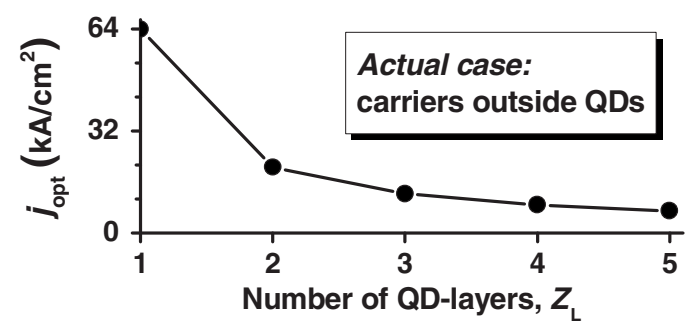

FIG. 4. Optimum dc injection current density maximizing the modulation bandwidth $\left(\omega_{-3 \mathrm{~dB}}^{\max } / 2 \pi=20 \mathrm{GHz}\right.$ at $\left.L=1.139 \mathrm{~mm}\right)$ vs number of QD-layers in the actual case of carriers outside QDs.

is $j_{\text {opt }}\left(64 \mathrm{kA} / \mathrm{cm}^{2}\right)$. Figure 4 shows the optimum de current density $j_{0}=j_{\text {opt }}$ maximizing $\omega_{-3 \mathrm{~dB}}$ versus number of QDlayers. While $j_{\text {opt }}$ is very high even for $Z_{\mathrm{L}}=2$, the use of four or five layers makes $\omega_{-3 \mathrm{~dB}}^{\max }$ practically attainable thus compensating for the adverse effect of carriers in the OCL.

In conclusion, we derived a closed-form expression for the upper limit for the modulation bandwidth of a QD laser. The highest possible bandwidth increases with increasing overlap integral of the electron and hole wave functions in a $\mathrm{QD}$, number of QD-layers and surface density of QDs in a layer, and with reducing QD-size dispersion. At 10\% QDsize fluctuations and $100 \%$ overlap, the upper limit for the bandwidth in a single QD-layer laser can be as high as 60 GHz.

L.V.A. acknowledges the U.S. Army Research Office (Grant No. W911-NF-08-1-0462) and R.A.S. acknowledges the Russian Foundation for Basic Research (Grant No. 0802-01337) for support of this work.

${ }^{1}$ Y. Arakawa and H. Sakaki, Appl. Phys. Lett. 40, 939 (1982).

${ }^{2}$ G. Park, O. B. Shchekin, D. L. Huffaker, and D. G. Deppe, IEEE Photonics Technol. Lett. 12, 230 (2000).

${ }^{3}$ P. G. Eliseev, H. Li, A. Stintz, G. T. Liu, T. C. Newell, K. J. Malloy, and L. F. Lester, Appl. Phys. Lett. 77, 262 (2000).

${ }^{4}$ I. C. Sandall, P. M. Smowton, C. L. Walker, H. Y. Liu, M. Hopkinson, and D. J. Mowbray, IEEE Photonics Technol. Lett. 18, 965 (2006).

${ }^{5}$ S. Fathpour, Z. Mi, P. Bhattacharya, A. R. Kovsh, S. S. Mikhrin, I. L. Krestnikov, A. V. Kozhukhov, and N. N. Ledentsov, Appl. Phys. Lett. 85 , 5164 (2004)

${ }^{6}$ T. Ikegami and Y. Suematsu, Proc. IEEE 55, 122 (1967).

${ }^{7}$ T. L. Paoli and J. E. Ripper, Proc. IEEE 58, 1457 (1970).

${ }^{8}$ M. J. Adams, Opto-electronics (London) 5, 201 (1973).

${ }^{9}$ R. F. Kazarinov and R. A. Suris, Sov. Phys. JETP 39, 522 (1974).

${ }^{10}$ C. B. Su and V. A. Lanzisera, IEEE J. Quantum Electron. 22, 1568 (1986).

${ }^{11}$ R. Olshansky, P. Hill, V. Lanzisera, and W. Powazinik, IEEE J. Quantum Electron. 23, 1410 (1987).

${ }^{12}$ R. Nagarajan, M. Ishikawa, T. Fukushima, R. S. Geels, and J. E. Bowers, IEEE J. Quantum Electron. 28, 1990 (1992).

${ }^{13}$ L. V. Asryan and R. A. Suris, Semicond. Sci. Technol. 11, 554 (1996).

${ }^{14}$ L. V. Asryan, M. Grundmann, N. N. Ledentsov, O. Stier, R. A. Suris, and D. Bimberg, J. Appl. Phys. 90, 1666 (2001).

${ }^{15}$ L. V. Asryan, S. Luryi, and R. A. Suris, IEEE J. Quantum Electron. 39, 404 (2003).

${ }^{16}$ N. N. Ledentsov, F. Hopfer, and D. Bimberg, Proc. IEEE 95, 1741 (2007). 